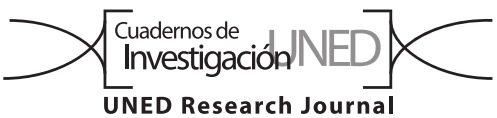

\title{
La enseñanza de la etnobiología a través de la educación a distancia evaluada desde el estudiantado en el período 2015-2016
}

\author{
Esteban Salazar-Acuña ${ }^{1}$ \\ Universidad Estatal a Distancia, Escuela de Ciencias Exactas y Naturales, San José, Costa Rica; esteban2085@gmail.com
}

Recibido 09-X-2018 • Corregido 22-I-2019 • Aceptado 30-I-2019

\begin{abstract}
Ethnobiology teaching in distance education evaluated for students". Introduction: ethnobiology as interdisciplinary science has had an important development in recent few decades in Latin America. In Costa Rica, the career of Natural Resource Management by State Distance University (UNED) is the only career that offers an Ethnobiology course at undergraduate level by a virtual mode. Objective: identify the strengths and weaknesses of the teaching of ethnobiology in Costa Rica through a virtual model of distance education; from the perception of undergraduate students of the Natural Resources Management career of the UNED in the 2015 and 2016 cohorts. Methods: the perception and quality of materials and educational resources applied were evaluated by 20 students belonging to the 2015 and 2016 cohorts through a survey. Results: I found that most of the students considered the subject highly positive in their academic education. The student was agreed that the use of ethnobiological tools is an important element in identification, classification of use and management of the natural resources by the communities and it can contribute to the conservation of those resources. Conclusion: However, ethnobiology is poorly incorporated into management programs in Costa Rica due to the limited knowledge of its application, not only in areas with indigenous populations, but in urban regions.
\end{abstract}

Key words: Ethnobiology, education, distance education model, perception, biodiversity, culture.
RESUMEN: Introducción: la etnobiología como ciencia interdisciplinaria ha tenido un importante desarrollo en las últimas décadas en América Latina, especialmente por su vinculación con los planes de conservación y su importancia en el manejo de los recursos naturales por parte de las comunidades. En el caso de Costa Rica, la carrera de Manejo de Recursos Naturales de la Universidad Estatal a Distancia (UNED) es única universidad del país que ofrece una asignatura de etnobiología en su nivel de licenciatura que ofrece por medio de una modalidad virtual. Objetivo: identificar las fortalezas y debilidades de la enseñanza de la etnobiología en Costa Rica a través de un modelo virtual de educación a distancia; desde la percepción de los estudiantes de nivel de licenciatura de la carrera de Manejo de Recursos Naturales de la UNED en las cohortes 2015 y 2016. Métodos: evalué la percepción de 20 estudiantes pertenecientes a las cohortes 2015 y 2016 a través de una encuesta acerca del impacto de la asignatura sobre su formación académica y profesional; así como la calidad en materiales y recursos didácticos aplicados. Resultados: encontré que la mayoría de los estudiantes consideraron de impacto positivo a la asignatura en su formación académica. Según los estudiantes, el uso de una plataforma virtual no limita la capacidad del desarrollo del proceso de enseñanza-aprendizaje. Conclusión: La etnobiología es poco estudiada e incorporada en los planes de manejo en Costa Rica debido al poco conocimiento de su aplicación, por lo que se debe lograr una mayor representación de la etnobiología en el currículo de las carreras asociadas a las ciencias naturales y sociales aplicadas.

Palabras clave: Etnobiología, educación a distancia, percepción, biodiversidad, cultura.

biológicas, ya que se estudian elementos naturales como la flora, fauna y recursos abióticos, por ejemplo; el suelo y el agua vinculados al conocimiento tradicional y conceptualización del universo (cosmovisión), lo que hace que sus métodos y aplicaciones sean multidisciplinarios entre las ciencias sociales y las ciencias naturales (Pretty et al., 2009).

Este conocimiento tradicional o ancestral está ligado a la utilización, manejo y conservación de los recursos naturales presentes en un sitio y es fundamental en la identificación de la "línea base" en la formulación de estrategias que procuren el mantenimiento de los servicios 
ecosistémicos como la disponibilidad de agua, tierras para cultivo, recursos para construcción, alimento, medicinas, entre otros; los cuales son conocidos y utilizados por las comunidades a partir del conocimiento generado a través del tiempo, de la misma forma que se promueve la protección y mantenimiento de la cultura local, así como su desarrollo social (Richeri, Ladio, \& Beekow, 2013).

Según De la Torre-Cuadros (2013), la aplicación del conocimiento local en los planes de conservación de la naturaleza ha aumentado en las últimas décadas, pero recopilado y aplicado de forma aislada a la información científica, por lo que ambas áreas no se relacionan con frecuencia o el conocimiento tradicional es omitido o subestimado

Pese a que el desarrollo de la etnobiología ha aumentado de forma significativa en el continente americano, la oferta académica de formación en esta área es limitada en las universidades (D’Ambrosio, 2012). En el caso de Costa Rica, la publicación científica en revistas indexadas sobre el tema es de las más escasas del continente (Albuquerque et al., 2013), lo que puede considerarse como un reflejo de los pocos profesionales, estudiantes y académicos que conocen, aplican o utilizan la etnobiología en su desarrollo profesional.

Esta realidad se ve reflejada en la oferta académica costarricense en temas directamente etnobiológicos. En la actualidad, la formación en etnobiología de profesionales relacionados con las ciencias naturales y aplicadas en la educación superior formal es optativa y limitada, por lo que la cantidad de estudiantes que tienen acceso a conocer y aplicar técnicas etnobiológicas en la conservación y manejo de los recursos en los niveles de pregrado y grado no supera a los 20 estudiantes por año. Existe oferta académica en postgrados públicos o en cursos especializados brindados por organizaciones privadas que ofertan estos cursos de forma aleatoria, como por ejemplo los brindados por Luis Diego Gómez para la Organización para Estudios Tropicales (Morera, 2010) y en cursos regionales promovidos por la Red Latinoamericana de Botánica entre el 2008 y el 2011 (Lagos-Witte et al., 2011) que estuvieron sujetos a la capacidad de los estudiantes de costear dicha capacitación o a los programas de becas que ofrecieron estas organizaciones.

En este contexto, la Universidad Estatal a Distancia (UNED) ofrece desde el 2015 dentro de su currículo optativo en la carrera de Licenciatura en Manejo de Recursos Naturales la asignatura "Etnobiología" para los estudiantes avanzados de dicho programa de estudios, como la única oferta nacional a nivel de pregrado y grado (Bachillerato y Licenciatura universitaria) disponible en la oferta universitaria pública, la cual fue incluida como asignatura optativa después de un proceso de rediseño de la malla curricular en el 2014, en donde se identificó la necesidad de contar con formación en etnobiología y antropología, así como realizar mejoras curriculares en otras asignaturas ya existentes en la malla (UNED, 2019). El modelo de educación a distancia como una forma de alcanzar a la población que por diversos motivos no puede integrarse a la educación presencial, es un desafío en cuanto a la planificación, metodologías, técnicas y herramientas (Sabogal, 2010). Es así como la enseñanza de la etnobiología en una modalidad de educación a distancia es un proceso nuevo, que posee pocos elementos para su evaluación y sistematización de los contenidos de aprendizaje, lo que hace fundamental generar una base de conocimiento sobre el abordaje y el desarrollo de educación superior en etnobiología bajo este esquema académico.

El objetivo de este trabajo fue identificar las fortalezas y debilidades de la enseñanza de la etnobiología en Costa Rica a través de un modelo virtual de educación a distancia; desde la percepción de los estudiantes de nivel de licenciatura de la carrera de Manejo de Recursos Naturales de la UNED en las cohortes 2015 y 2016.

\section{MATERIALES Y MÉTODOS}

Estructura curricular y modelo de enseñanza-aprendizaje de la asignatura "Etnobiología" de la UNED: Etnobiología es una asignatura electiva formulada para el nivel de Licenciatura de la carrera de Manejo de Recursos Naturales de la Escuela de Ciencias Exactas y Naturales, la cual fue incorporada en el plan de estudios en la modificación de la malla curricular de la carrera en el 2014. La carga académica asignada es de tres créditos universitarios, lo que implica una inversión de 135 horas para los estudiantes, distribuidos en un período cuatrimestral (setiembre a diciembre).

EL objetivo general de la asignatura es "Valorar las relaciones del hombre con los seres vivos de su ambiente, mediante la determinación del papel que desempeñan las plantas y los animales en diversas sociedades y culturas" (UNED, 2018, p.3).

Al tratarse de una carrera principalmente fundamentada en las ciencias naturales, el enfoque de la asignatura es ecológico (Benetti, Vieira, Crepaldi, \& Ribeiro, 2013); por lo que se enfatiza en las relaciones humano-naturaleza, con un énfasis de estas relaciones sobre las poblaciones y ecología de los organismos, así como la capacidad de evaluar, entender y aplicar acciones que permitan la conservación de la diversidad biocultural. 
Toledo \& Barrera-Bassols (2008) definieron este abordaje biocultural como el estudio de los cambios biológicos, lingüísticos, cognitivos, agrícolas y paisajísticos relacionados con el ser humano en su entorno a través del tiempo, facilitados por su alta capacidad de adaptación a nuevos entornos y modificarlos para su beneficio.

En este contexto, según la orientación académica de la asignatura (UNED, 2018, p. 3), los objetivos específicos planteados en la asignatura son:

- Describir los conceptos básicos relacionados con la etnobiología.

- Relacionar las actividades del ser humano con su ambiente y sus implicaciones en la conservación de la diversidad biológica y cultural.

- Comparar los diferentes usos, la valorización y el aprovechamiento de los recursos naturales en diferentes sociedades.

- Analizar la importancia de la diversidad cultural, biológica y el papel del conocimiento tradicional para la conservación de los recursos naturales.

Instrumentos de evaluación aplicados en la asignatura: encuentra conformada por cuatro tipos de instrumentos que emplearon en la evaluación de los aprendizajes en las cohortes 2015 y 2016, los cuales fueron las 1) "Tareas" o trabajo de ejecución, 2) foros de discusión, 3) Giras y sus informes de campo y 4) el trabajo final de ejecución (estudio de caso o investigación dirigida).

En las cohortes estudiadas, los instrumentos de evaluación mantuvieron el mismo formato básico, sin embargo, entre el 2015 y el 2016, los instrumentos fueron diferentes en cuanto a las preguntas solicitadas a los estudiantes, de manera que no se repitieran los enunciados de los instrumentos entre cohortes. No obstante, los objetivos de aprendizaje fueron los mismos en ambos años.

En el desarrollo de las tareas, los estudiantes tenían que realizar investigación y descripción de conceptos básicos en etnobiología y su aplicación en el manejo de los recursos naturales; así como desarrollar ejercicios básicos sobre cálculo de índices matemáticos de uso común en los trabajos etnobiológicos, siguiendo la lista de índices descrita por Alburquerque, Cruz Da Cuhna, Paiva de Lucena, y Nóbrega-Alves (2014).

Los foros académicos se basaron en la generación de espacios de discusión de temas específicos moderados por el tutor del curso, en donde los estudiantes deben presentar y argumentar ideas, conclusiones o hipótesis fundamentadas en sus experiencias profesional o académicas, respaldadas en literatura en etnobiología. Cada estudiante tuvo espacio de participación en dos foros académicos con posibilidad de ingresar tres comentarios por foro, uno de inicio hacia el comentario generador que realiza el tutor y dos adicionales comentando el aporte de otros alumnos. Al final de cada foro, el tutor realiza una retroalimentación de los comentarios y aportes de cada estudiante. Los foros utilizan recursos audiovisuales como documentales cortos o vídeos como elemento de referencia en la discusión que modela el tutor de la asignatura.

En tercer lugar, las giras de campo se evaluaron mediante dos instrumentos complementarios. El primer instrumento aplicado fue la evaluación in situ que realizó el tutor sobre cada uno de los estudiantes durante el desarrollo de las giras y el segundo instrumento fue el informe de gira que cada estudiante entregó posterior a cada actividad de campo. La asignatura realiza dos giras de campo, la primera con duración de diez horas y la segunda con una duración de 48 horas. Los lineamientos de evaluación son entregados a los estudiantes previo al inicio de las giras.

Por último, el trabajo final consistió en un ejercicio teórico-práctico grupal en donde los estudiantes desarrollar un proyecto de investigación en donde se busca aplicar todos los métodos y técnicas estudiadas en el curso. Este trabajo es la última actividad programada de la asignatura. En el 2015 el tema desarrollado consistió en una evaluación etnobiológica de los jardines familiares en un gradiente de urbanización en el Valle Central y en el 2016 se desarrolló una investigación dirigida sobre la venta y comercialización de plantas medicinales en los cuatro mercados principales del Valle Central del país.

Percepción de los estudiantes acerca del impacto y calidad del curso: Para identificar la percepción de los estudiantes acerca del impacto de la asignatura en su formación, se aplicó un instrumento tipo encuesta estructurada a los estudiantes de las cohortes de los años 2015 y 2016. Este instrumento contó con 18 preguntas enfocadas en recopilar la percepción de los estudiantes acerca de la calidad de la asignatura, los recursos brindados, la preparación del docente y la percepción del impacto del curso en la formación profesional en el área del manejo de recursos naturales y las áreas que los estudiantes consideraron como prioritarias para que sean abordadas en asignaturas posteriores vinculadas a la etnobiología, además de la percepción general de los instrumentos metodológicos aplicados durante el desarrollo del curso. Se utilizó una escala Likert de 5 categorías desde un 
valor mínimo de 1 (Deficiente) hasta un valor máximo de 5 (Muy alta).

La validación del instrumento se realizó por medio del criterio experto de la Cátedra de Ecología y Educación Ambiental de la Escuela de Ciencias Exactas y Naturales de la UNED antes de su aplicación a los estudiantes.

El instrumento de evaluación se aplicó a través de la plataforma Google forms y fue enviado a los estudiantes de las cohortes estudiadas después de finalizado el proceso académico en su respectivo período académico. En total se envió el instrumento a los 33 estudiantes que cursaron la asignatura entre el 2015 y el 2016.

Para la evaluación del cumplimiento de los objetivos y la calidad de la asignatura como proceso académico, dentro del mismo instrumento se encuestó a los estudiantes acerca de la calidad de los materiales y recursos didácticos utilizados, la organización de la plataforma virtual y los instrumentos de evaluación aplicados. Se utilizó una escala Likert de 5 categorías desde un valor mínimo de 1 (Deficiente) hasta un valor máximo de 5 (Excelente). Las respuestas recibidas fueron comparadas entre cohortes por medio de un análisis chi-cuadrado para determinar si existieron diferencias entre el año 2015 y 2016.

Ética, conflicto de intereses y declaración de financiamiento: el autor declara haber cumplido con todos los requisitos éticos y legales pertinentes, tanto durante el estudio como en el manuscrito; que no hay conflictos de interés de ningún tipo. Asimismo, está de acuerdo con la versión editada final del documento. El respectivo documento legal firmado se encuentra en los archivos de la revista.

\section{RESULTADOS}

La asignatura etnobiología se imparte a través de una plataforma virtual, por lo que se ha logrado que tanto estudiantes de zonas urbanas como rurales tengan acceso. Entre el 2015 y el 2016 la matrícula total fue de 33 estudiantes de 11 Centros Universitarios (CU), de los cuales seis se ubican en zonas rurales. El $80 \%$ de los estudiantes fueron mujeres y el $20 \%$ hombres.

La distribución de CU en donde se encontró representación de los estudiantes de la Carrera de Manejo de Recursos Naturales en las cohortes 2015 y 2016, permite establecer que el alcance geográfico es inclusivo, ya que la oferta del curso estuvo disponible a nivel nacional. Sin embargo, no se pudo establecer representación estadística de la población estudiantil; ya que el curso es de carácter optativo.
Percepción de los estudiantes sobre el impacto del curso en la formación académica y profesional: El $77 \%$ de los estudiantes tuvieron como formación base (pregrado) la carrera de manejo de recursos naturales, mientras que el restante 33\% poseían como formación base biología e ingeniería forestal. Todos los estudiantes encuestados fueron bachilleres universitarios egresados (pendientes del acto graduación) o graduados.

La encuesta de percepción fue enviada a los 33 estudiantes que conformaron las cohortes 2015 y 2016 del curso; de los cuales 20 estudiantes respondieron. De estos estudiantes, el 50\% correspondió a la cohorte 2015 y el 50\% restante correspondió a la corte del 2016.

Se encontró que la intención principal de los estudiantes para cursar la asignatura respondió en un 45\% ( 9 estudiantes) a que no se tenía conocimiento sobre el concepto de etnobiología, un 35\% (7 estudiantes) de los estudiantes matricularon el curso ya que los contenidos generaron interés y solamente un $5 \%$ de los encuestados conocía el concepto previamente.

En el caso del impacto de la formación recibida sobre el desarrollo profesional, se encontró que 18 estudiantes consideraron que el curso impactó positivamente sobre su formación profesional. El 85\% (17 estudiantes) indicó que estaría dispuesto a cursar otra asignatura relacionada con la etnobiología mediante un sistema de educación a distancia.

Por último, al consultar si existía interés por parte de los estudiantes en la continuación de la oferta académica vinculada a la etnobiología; se encontró que existió un alto porcentaje de los estudiantes que mostraron interés en las áreas de etnoecología y en etnobotánica, sin embargo, mostraron un interés considerable (más del 50\%) en que se oferten cursos específicos de etnozoología y en menor medida en botánica económica (Fig. 1). No se encontró diferencia significativa entre las respuestas de ambas cohortes $\left(x^{2}=4,80 ;\right.$ g.l. $\left.=2 ; p<0,05\right)$.

Por último, el $90 \%$ de los estudiantes afirmó que la etnobiología debería abordarse de una forma más amplia dentro del currículo del programa de Manejo de Recursos Naturales, mientras que solamente el 5\% (un estudiante) no mostró interés en ampliar su conocimiento en la temática.

Percepción de los estudiantes sobre el desarrollo del proceso de enseñanza-aprendizaje.: Se encontró que la mayoría de los estudiantes consideraron que los recursos didácticos, las herramientas digitales (plataforma virtual y sus contenidos) y los instrumentos de evaluación fueron adecuados para el desarrollo del proceso 


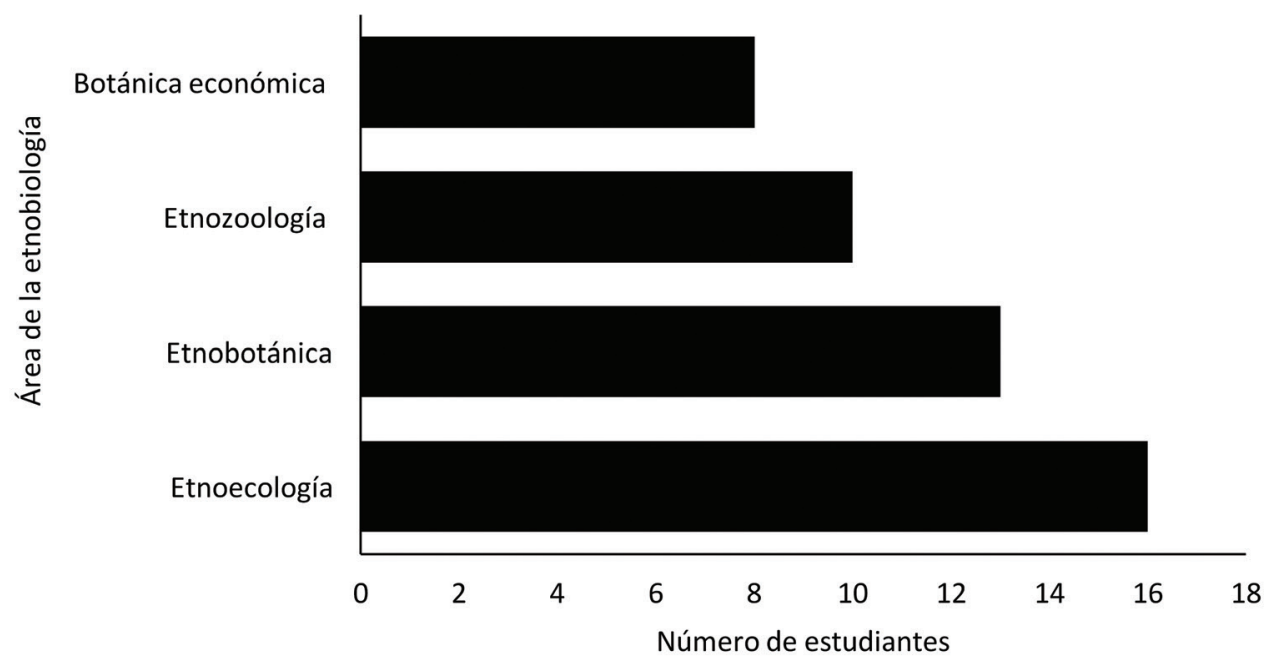

Fig. 1. Áreas temáticas de interés en profundizar formación académica de los estudiantes de la asignatura "etnobiología" en las cohortes 2015 y 2016.

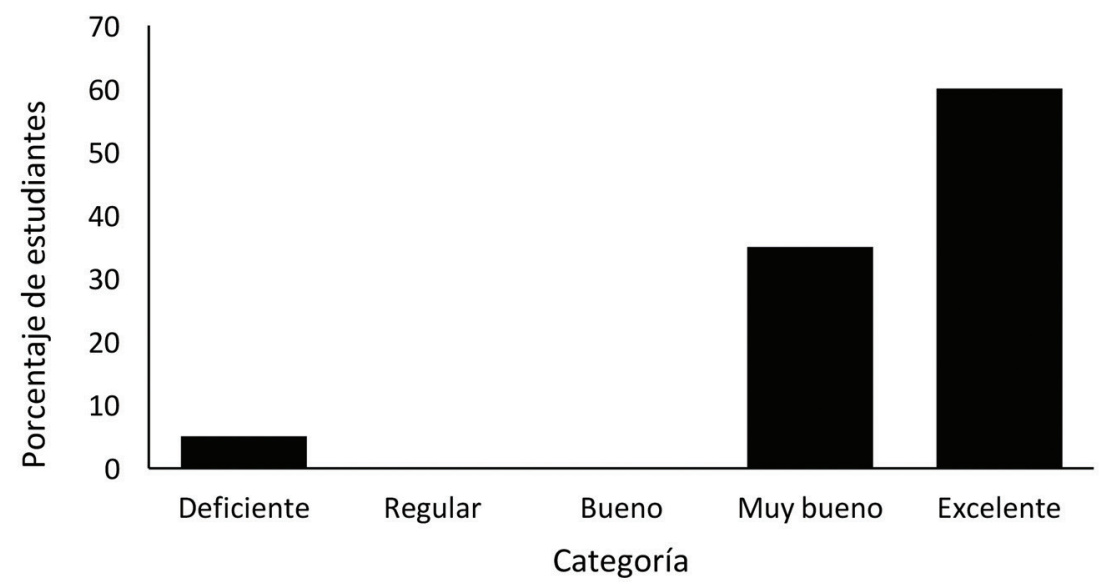

Fig. 2. Percepción de los estudiantes acerca de la calidad de los recursos didácticos utilizados den la asignatura Etnobiología en las cohortes 2015 y 2016.

de enseñanza-aprendizaje. No se encontró diferencia entre la percepción de las cohortes 2015 y $2016\left(x^{2}=5,38\right.$; $\mathrm{g} . \mathrm{l}=2 ; \mathrm{p}<0,05)$. En el caso de los recursos didácticos, el $60 \%$ de los encuestados consideraron como "Excelentes" y el $35 \%$ lo consideraron "Muy bueno". Solamente el 5\% consideró a la plataforma como "Deficiente" (Fig. 2).

En cuanto a la preparación del tutor del curso, se encontró que el $70 \%$ de los estudiantes indicaron que la preparación del tutor fue "Excelente", mientras que el $20 \%$ indicó que la preparación fue "Muy buena" y solamente un 5\% indicó que fue "Regular" (Fig. 3). Ninguno de los encuestados indicó que el tutor fue de "Deficiente".

\section{DISCUSIÓN}

La etnobiología en los últimos años ha tomado una gran relevancia en los programas de conservación y manejo de los recursos naturales a través de la defensa de los territorios y los derechos de los pueblos indígenas sobre sus recursos y del conocimiento tradicional asociado, ya que la importancia de reconocer la percepción y el conocimiento de las comunidades acerca de los recursos que los rodean como un elemento de manejo, permite formular estrategias más integrales, aplicando nuevos modelos bioculturales (Amo-Rodríguez, 2011). Esta integración del componente etnobiológico desde 


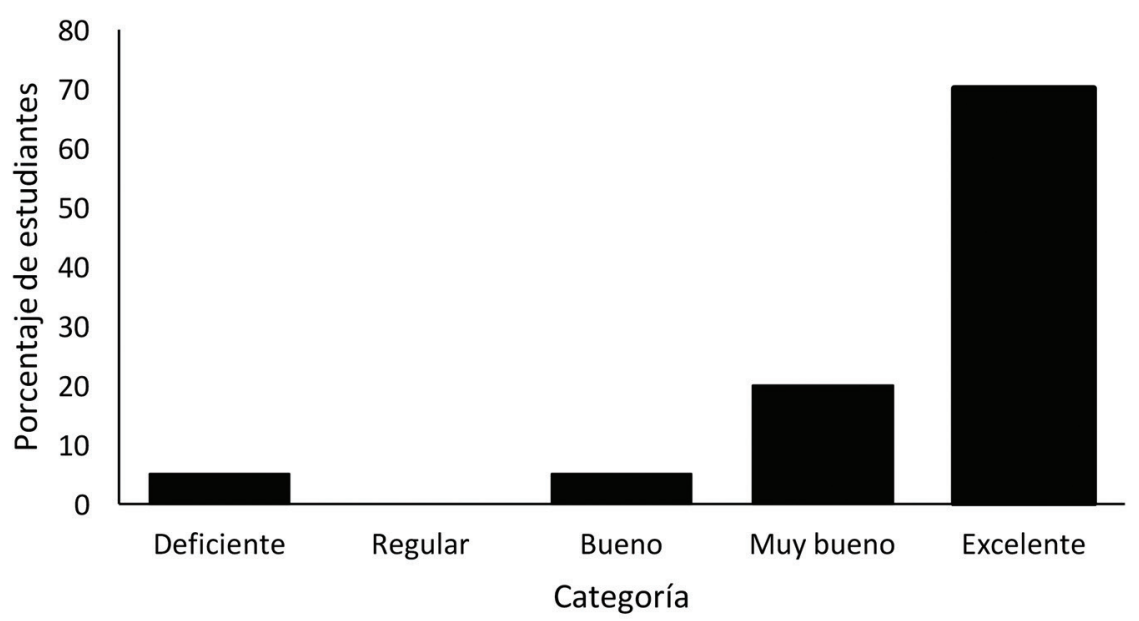

Fig. 3. Percepción de los estudiantes acerca de la preparación del tutor.

un enfoque multidisciplinario que ha permitido que los estudios sobre el conocimiento, la percepción y el uso de los recursos naturales bióticos y abióticos sea cada vez más frecuentes.

No obstante, desde hace muchos años, se realizan trabajos de investigación relacionados con el uso y manejo de los recursos naturales por diferentes comunidades y grupos étnicos, especialmente indígenas y campesinos. Sin embargo, a nivel nacional, el enfoque de estas investigaciones se puede catalogar como incipiente en las etapas del desarrollo de la etnobiología (Hunn, 2007).

La asignatura de etnobiología es impartida a través de una modalidad con un alto componente virtual, que responde a la necesidad de incorporar a las TIC en la educación a distancia (Méndez-Estrada \& Monge-Nájera, 2006), a través del uso y manejo de la plataforma en línea para el desarrollo del proceso de enseñanza-aprendizaje en casi la totalidad de sus actividades, en donde los estudiantes deben ingresar al entorno virtual para obtener los recursos, literatura, instrumentos e instrucciones de la asignatura con excepción de las giras de campo. Este abordaje a utilizado en otros sistemas de educación a distancia, ya que todo el acompañamiento, recursos y materiales se manejaron en la plataforma en línea.

Lo anterior propone un escenario complejo para la evaluación de una asignatura de etnobiología abordada virtualmente, ya que no se tiene experiencia previa en la educación de este tema superior bajo este modelo específico. No obstante, la medición del impacto de la etnobiología en la educación ya se ha abordado en educación formal y presencial a nivel de primaria y secundaria en países como Brasil (Boccasius, 2012; Baptista \& Niño, 2009), México (Azúa \& Téllez, 2012), Colombia (Vasco, 2004) y en las islas del Pacífico (McCarter \& Gavin, 2011); en donde la aplicación de la etnobiología en educación requiere de un cambio profundo en el sistema educativo (Vargas-Clavijo, Medeiros, \& Costa, 2014). En Costa Rica, la etnobiología en la educación superior formal es un tema de desarrollo incipiente, especialmente en los niveles básicos (grada y pregrado) por lo que la divulgación y documentación de experiencias con modelos educativos no aplicados con anterioridad es fundamental para el desarrollo de nuevas herramientas y procesos de enseñanza-aprendizaje.

A nivel de postgrado si existen algunas experiencias específicas en la Universidad de Costa Rica (UCR), La Organización de Estudios Tropicales (OET) y el Centro Agronómico de Investigación y Enseñanza (CATIE), que han contribuido con el desarrollo de la etnobiología costarricense. Sin embargo, al encontrarse en los niveles avanzados de la formación o en postgrados, si existe una limitación en cuanto al acceso general de los estudiantes al tema.

La poca experiencia que se tiene en la incorporación de la etnobiología en el currículo formal de la educación superior en Costa Rica, no como complemento en contenidos en las áreas de botánica, zoología o ecología, sino como una asignatura dentro de la formación académica de profesionales en ciencias naturales y aplicadas, hace complejo establecer líneas de éxito en la ejecución y cumplimiento de los objetivos de la asignatura. No obstante, la alta cantidad de los estudiantes encuestados que muestran que los contenidos desarrollados en la asignatura fueron identificados como de alta relevancia por los alumnos.

La baja posibilidad para los estudiantes de ciencias naturales y sociales del país para acceder a una oferta académica en etnobiología podría representar una de 
las causas de que Costa Rica sea uno de los países con menor desarrollo de publicaciones indexadas en el tema en los últimos años (Albuquerque et al., 2013). Es importante recalcar que Costa Rica posee aportes importantes en la etnobiología latinoamericana por parte de investigadores como Rafael Ocampo, Luis Poveda Álvarez, Luis Diego Gómez y la investigadora hondureña Sonia LagosWitte, entre otros. Sin embargo, no existe una estructura académica formal que facilite la transición generacional en la investigación y enseñanza de la etnobiología en la academia costarricense.

Existen algunos autores que presentan trabajos recientes desarrollados en Costa Rica sobre plantas medicinales (García-González \& Morales 2005; Contreras \& Campregher, 2010), el uso de plantas en jardines urbanos (González-Balí, Bermúdez-Rojas, \& Romero-Vargas, 2017), la percepción de las comunidades acerca de algunos grupos faunísticos (Valdelomar et al., 2012; Rocha \& Salazar, 2004), que demuestran que la etnobiología no está ausente en Costa Rica, pero que, en comparación con otros países de la región, la generación de información local en los últimos 15 años es escasa.

Este bajo desarrollo de investigación y publicación en Costa Rica con respecto a la región latinoamericana puede estar vinculada a diversos factores internos y externos de la academia. Entre los principales factores que se pueden identificar la falta de información que existe en Costa Rica acerca de la aplicación de elementos etnobiológicos en el estudio, planificación del manejo y conservación de los recursos naturales y culturales. Además, la falta de reconocimiento de la etnobiología como ciencia por parte de algunos sectores de la comunidad científica debido a diversos factores históricos y de conformación de la disciplina científica (Pretty et al., 2009), la escasa población indígena identificada en el país (alrededor del $2 \%$ de la población), ubicada en su mayoría en áreas alejadas a los centros de alta densidad poblacional del país (MIDEPLAN, 2015), los pocos miembros que conforman sociedad o grupos académicos formales (Wyndham, Lepofsky, \& Tiffany, 2011), entre otros.

En el caso del desarrollo del proceso de enseñanza como un proceso académico, la encuesta realizada a los estudiantes muestra que los estudiantes consideran que los materiales y recursos utilizados en el curso en su mayoría, permiten el desarrollo de las actividades académicos sin comprometer la calidad en la enseñanza de la asignatura, por lo que se puede establecer que la percepción de los estudiantes es positiva en cuanto a la formación recibida a través de un modelo de educación a distancia y en una modalidad virtual. Es importante considerar que los estudiantes evaluados son profesionales graduados o en proceso de graduación; por lo que su formación alcanzada puede permitir suponer un mayor grado de análisis del impacto en su formación académica de la asignatura que en niveles más tempranos.

La etnobiología como ciencia multidisciplinaria permite la identificación y propuestas de solución a problemas de manejo específicos, relacionados principalmente con las relaciones culturales entre los seres humanos y los organismos vivos en su entorno, principalmente vinculados con el uso, manejo, domesticación, cultivo y consumo de plantas, animales, hongos y otros organismos. Además, su aplicación permite no solamente abordar problemáticas en áreas con comunidades indígenas, sino urbanas o semi-urbanas, como por ejemplo los trabajos realizados por Pochettino, Arenas, Sánchez, y Correa (2008) en Argentina; Albuquerque, Monteiro, Ramos, y Amorim (2007) en Brasil; Marcia, García, y Vidaurre (2005) en Bolivia y Cuvi (2017) en Ecuador, entre otros.

Por último, la percepción del estudiantado acerca del desempeño de una asignatura no es la única variable que se debe incluir en una evaluación educativa, por lo que es importante que, si se evalúa el alcance de dicha asignatura de forma sistemática, se deben incluir variables como su aplicación en el desarrollo profesional, el avance en la investigación de temas etnobiológicos a escala nacional, la incorporación de nuevas asignaturas en el currículo y los resultados de los procesos internos de evaluación de cada una de elementos del proceso de enseñanza-aprendizaje; por lo que este trabajo debe complementarse con otras variables de evaluación.

La experiencia exitosa en el desarrollo de la asignatura de etnobiología ha permitido identificar la necesidad de promover más formalmente el estudio y la investigación etnobiológica en Costa Rica, especialmente aplicada al manejo de los recursos naturales, no solamente en comunidades indígenas o campesinas; sino en áreas urbanas, en los mercados locales y en ferias del agricultor, entre otros. Es necesario generar información acerca del desempeño y alcances de la formación académica formal en etnobiología en el país, así como la capacidad de extender el proceso de enseñanza a la aplicación en la función profesional y en la generación de investigación.

\section{AGRADECIMIENTOS}

Un agradecimiento especial a la Escuela de Ciencias Exactas y Naturales de la Universidad Estatal a Distancia, específicamente a Idannia Valverde Rojas por toda su colaboración y apoyo en el desarrollo de la asignatura y en su mejoramiento constante. 
A los estudiantes del curso de las cohortes 2015 y 2016 por su apoyo en el llenado del instrumento de percepción de la asignatura, con sus comentarios y recomendaciones.

A Olga Lucía Sanabria Diago de la Universidad del Cauca y Álvaro Sepúlveda de la Sociedad Colombiana de Etnobiología (SCE), por todas sus recomendaciones y consejos en la elaboración de este trabajo.

\section{REFERENCIAS}

Alburquerque, U., Cruz Da Cuhna, L., Paiva de Lucena, R., \& Nóbrega-Alves (eds.). (2014). Methods and Techniques in Ethnobiology and Ethnoecology. Springer Protocols Handbooks. New York: Humana Press. DOI: 10.1007/978-1-4614-8636-7

Albuquerque, U., Monteiro, J., Ramos, M., \& Amorim, C. (2007). Medicinal and magic plants from a public market in northeastern Brazil. Journal of Ethnopharmacology, 110, 7691. DOI: 10.1016/j.jep.2006.09.010

Albuquerque, U., Soares-Silva, J., Loureiro, J., Silva, R., Silva, T., \& Nóbrega, R. (2013). The current status of ethnobiological research in Latin America: gaps and perspectives. Journal of ethnobiology and ethnomedicine, 9, 72. DOI: 10.1186/1746-4269-9-72

Amo-Rodríguez, S. (2011). El cambio de modelo de desarrollo necesario en un país pluricultural. algunos aspectos que se deben tomar en cuenta. Etnobiología, 9, 60-76.

Azúa, R., \& Téllez, M. (2012). Cosmos, Corpus y Praxis de le etnobiología mexicana aplicado en la educación básica. Etnobiología, 10(1), 52-59.

Baptista, G., \& Niño, C. (2009). The contribution of Ethnobiology to the Construction of a Dialogue Between Ways of Knowing: A case studying a Brazilian Public High School. Science \& Education, 18(3-4), 503-520. DOI: 10.1007/ s11191-008-9173-3

Benetti, I., Vieira, M., Crepaldi, M., \& Ribeiro, D. (2013). Fundamentos da teoria bioecológica de Urie Bronfenbrenner. Pensado Psicologia, 9(16), 89-99. DOI: 10.16925/pe.v9i16.620

Boccasius, A. (2012). Etnobiología en la educación básica. Revista de Educación en Biología, 15(2), 12-19.

Clément, D. (1998). The historical foundations of ethnobiology (1860-1899). Journal of ethnobiology, 18(2), 167-187.

Contreras, A., \& Campregher, C. (2010). Plantas medicinales en una plantación de cacao en Guápiles, Costa Rica. UNED Research Journal, 2(2). 231-237. DOI: 10.22458/urj. v2i 2.159

Cuvi, N. (2017). Las ciudades como mosaicos bioculturales: el caso del centro histórico de Quito. Etnobiología, 15(1), $5-25$.
D’Ambrosio, U. (2012). Theoretical reflections on ethnobiology in the third millennium. Contributions to Science, 10, 49-69.

De la Torre-Cuadros. M. (2013). Nota científica: hacia un enfoque biocultural en los programas de conservación de la naturaleza. Etnobiología, 11(1), 53-57.

García-González, M., \& Morales, C. (2005). Análisis de literatura sobre plantas medicinales en Costa Rica (1930-2001). Lankesteriana, 5(1), 3-40.

González-Balí, R., Bermúdez-Rojas, T., \& Romero-Vargas, M. (2017). Floristic composition and richness of urban domestic gardens in three urban socioeconomic stratifications in the city Heredia, Costa Rica. Urban Ecosystems, 20(1), 51-63. DOI: 10.1007/s11252-016-0587-4

Hunn, E. (2007). Ethnobiology en four phases. Journal of Ethnobiology, 27(1), 1-10. DOI: 10.2993/0278-0771(2007)27[1:EIFP]2.0.CO;2

Marcia, M., García, E., \& Vidaurre, P. (2005). An ethnobotanical survey of medicinal plants commercialized in markets of La Paz and El Alto, Bolivia. Journal of Ethnopharmacology, 97, 337-350. DOI: 10.1016/j.jep.2004.11.022

McCarter, J., \& Gavin, M. (2011). Perceptions of the value of traditional ecological knowledge to formal school curricula: opportunities and challenges from Malekula Island, Vanuatu. Journal of Ethnobiology and Ethnomedicine, 7, 38. DOI: 10.1186/1746-4269-7-38

Medeiros, E. (2011). Etnobiología y el proceso de empoderamiento de los pueblos tradicionales. Ecología en Bolivia, 46(1), 1-3.

Méndez-Estrada, V., \& Monge Nájera, J. (2006). Las TIC en un entorno latinoamericano de educación a distancia: la experiencia de la UNED en Costa Rica. Revista de Educación a Distancia, 15, digital. Recuperado de http://www. um.es/ead/red/15.

MIDEPLAN (Ministerio de Planificación Nacional y Política Económica). (2015). Población Indígena en cifras. San José, Costa Rica.

Morera, P. (2010). In Memoriam: Luis Diego Gómez Pignataro 1944-2009. Revista de Biología Tropical, 58(4), XIX-XXI.

Lagos-Witte, S., Sanabria, O., Chacón, P., \& García, R. (2011). Manual de herraamientas etnobotánicas relativas a la conservación y el uso sostenible de los recursos vegetales. Una contribución de la Red Latinoamericana de Botánica a la implementación de la Estrategia Global de Conservación de Especies Vegetales hacia el logro de las metas 13 y 15. Red Latinoamericana de Botánica. Santiago, Chile.

Pochettino, M., Arenas, P., Sánchez, D., \& Correa, R. (2008). Conocimiento botánico tradicional, circulación comercial y consumo de plantas medicinales en un área urbana de Argentina. Boletín Latinoamericano de Plantas Medicinales y Aromáticas, 7(3), 141-148. 
Pretty, J., Adams, B., Berkes, F., Ferreira de Athayde, S., Dudley, N., Hunn, E., Maffi, L., Milton, K., Rapport, D., Robbins, P., Sterling, E., Stolton, S., Tsing, A., Vintinner, E., \& Pilgrim, S. (2009). The Intersections of Biological Diversity and Cultural Diversity: Towards Integration. Conservation and Society, 7(2), 100-112. DOI: 10.4103/0972-4923.58642

Richeri, M., Ladio, A., \& Beekow, A. (2013). Conocimiento tradicional y autosuficiencia: la herbolaria rural en la Meseta Central del Chubut (Argentina). Boletín Latinoamericano y del Caribe de Plantas Medicinales y Aromáticas, 12(1), 44-58.

Rocha, P., \& Salazar, J. (2004). Conocimiento popular sobre los búhos en los alrededores de un bosque húmedo tropical protegido en Costa Rica. Etnobiología, 4, 41-53

Sabogal, A. (2010). Modelos de educación a distancia. Revista de investigaciones UNAD Bogotá, 9, 223-230. DOI: $10.22490 / 25391887.727$

Toledo, V., \& Barrera-Bassols, N. (2008). La memoria biocultural. La importancia ecológica de las sabidurías tradicionales. Barcelona, España: Editorial Icaria.

UNED (Universidad Estatal a Distancia). (2018). Orientación Académica de la Asignatura Etnobiología 03227. Escuela de Ciencias Exactas y Naturales. Universidad Estatal a Distancia, San José, Costa Rica.

UNED (Universidad Estatal a Distancia). (2019). Cursos electivos. Escuela de Ciencias Exactas y Naturales. Recuperado de: https://www.uned.ac.cr/ecen/carrera/manejo-de-recursos-naturales/catedras-del-programa\#catedra-ecologia-y-educacion-ambiental-cod-202).

Valdelomar, V., Ramírez-Vargas, M., Quesada-Acuña, S., Arrieta, C., Carranza, I., Ruíz-Morales, G., Espinoza, S., MenaVillalobos, J., Brizuela, C., Miranda-Fonseca, L, MatarritaHerrera, M., González, J., Calderón-Sancho, E., Araya, J., Sauma-Rossi, A., Sandoval, I., \& Gómez-Lepiz, A. (2012). Percepción y conocimiento popular sobre el cocodrilo Crocodrylus acutus (Reptilia: Crocodylidae) en zonas aledañas al Río Tempisque, Guanacaste, Costa Rica. UNED Research Journal, 4(2), 191-202.

Vargas-Clavijo, M., Medeiros, E., \& Costa, G. (2014). De la superioridad de los currículos de biología al diálogo intercultural en la enseñanza de las ciencias. Etnobiología, 12(3), 17-27.

Vasco, L. (2004). Etnoeducación y etnobiología: ¿Una alternativa? Acta Biológica Colombiana, 9(2), 67-70.

Wolverton S., Kimberlee, J., \& Veteto, J. (2014). Climate Change and Ethnobiology. Journal of ethnobiology, 34(3), 273275. DOI: 10.2993/0278-0771-34.3.273

Wyndham, F., Lepofsky, D. \& Tiffany, S. (2011). Taking stock in ethnobiology: where do we come from? what are we? where are we going? Journal of ethnobiology, 31(1), 110127. DOI: 10.2993/0278-0771-31.1.110 